\title{
Optimization Model for Multi-Vehicle-Type Structure in Urban Bus Systems
}

\author{
Shuzhi Zhao, ${ }^{1}$ Dong Wang, ${ }^{1}$ Jian Sun, ${ }^{2}$ and Huasheng Liu ${ }^{1}$ \\ ${ }^{1}$ School of Transportation, Jilin University, Changchun 130022, China \\ ${ }^{2}$ School of Automobile, Chang'an University, Xian 170016, China \\ Correspondence should be addressed to Huasheng Liu; jtliuhs@jlu.edu.cn
}

Received 8 December 2016; Revised 15 March 2017; Accepted 27 March 2017; Published 9 April 2017

Academic Editor: Hakim Naceur

Copyright (c) 2017 Shuzhi Zhao et al. This is an open access article distributed under the Creative Commons Attribution License, which permits unrestricted use, distribution, and reproduction in any medium, provided the original work is properly cited.

\begin{abstract}
Nowadays, bus lines commonly share terminals, especially in urban areas, because an independent operation of each line would cause a large waste of resources. In addition, if lines share terminal all lines can be monitored by unified management, while the vehicles of different sizes can complement each other. Although the dispatcher generally sets up fixed departure intervals for bus lines, there are differences between bus lines in terms of operating time distribution and line volume. Thus, a new optimization model for multi-vehicle-type structure in urban bus system is proposed in this paper. The optimization was conducted according to two main characteristics of bus systems, bus company expense and bus service quality. The optimization model was designed based on minimal possible expense and maximal possible quality. At the same time, we proposed a Markov method based on alternative relations to solve the model. The proposed model was verified by real urban bus system. Moreover, the simulations in MATLAB software environment have proven a high efficiency and applicability of the proposed method.
\end{abstract}

\section{Introduction}

A proper configuration of multi-vehicle-type structure and bus timetable in urban bus systems represents comprehensive research topic. As it is well known, public transportation represents a public benefit, and its main purpose is to satisfy the travel needs of residents. Although the public transportation has been studied for a long time, it still needs some improvements. Ferland and Michelon [1] defined the public transport problem and showed that heuristic and exact methods, developed for scheduling with time windows and single type of vehicles, can be applied on a multiple-vehicletype structure. Moreover, Ceder [2] used empty trips to adjust the departure interval in order to arrange the lines. However, Ceder considered transport system with different vehicle types, wherein the vehicles were arranged according to their type, price, and quality. Ker et al. [3] used different sizes of vehicles on the same bus line and determined the optimal vehicle size for different time periods. In addition, in order to minimize the operating cost and passengers' travel cost, they develop the formula for calculation of a proper vehicle size. Site and Filippi [4] presented an optimal bus service model for urban areas and optimized the lines scheduling for different operation modes, such as a multivehicle mode. In [5] Zhu et al. studied the bus line capacity and analyzed the factors that influence the bus line equipment. Namely, using the restrictions of bus line length, route turnover, and departure interval, they calculated the number of vehicles based on bus types and road conditions. Kliewer et al. [6] discussed the multidepot multi-vehicle-type bus scheduling problem (MDVSP) considering multiple depots and different vehicle types for timetabled trips. In addition, MDVSP modeling presented in [6] was based on timespace-based networks instead of connection-based networks. In order to calculate the best size for passenger-carrying vehicles, dell'Olio et al. [7] proposed a bilevel optimization model with constraints on bus capacity, which allows buses of different sizes to be assigned to public transport routes and optimizes the headways on each route in accordance with observed demands. At the upper level, model optimizes social and operating cost of system, which represents the sum of user cost and operator cost. At the lower level, an assignment model for public transport with constraints on 
vehicle capacity balances bus size and headways flow in each iteration. Sun et al. [8] used the heuristic algorithm to develop a flexible timetable optimization method based on hybrid vehicle size model to tackle the bus demand fluctuations in transit operation. Huang et al. [9] studied the public multi-vehicle transportation systems and constructed the model for balanced passenger rate based on optimization of departure intervals and different bus sizes. Moreover, the proposed model makes departure interval and maximum capacity of variation uniform. Finally, the suggested optimization model uses the heuristic algorithm and provides the case analysis. Based on the mathematical model of multivehicle distribution path optimization problem, Xiong and $\mathrm{Hu}$ [10] obtained the optimal vehicle scheduling method based on minimal fuel consumption as the objective function of genetic algorithm. Huo [11] studied the vehicle structure optimization and analyzed its influencing factor, vehicle type, and technical parameters. Furthermore, the author established an improved model based on Markov forecast method and verified it by vehicle structure optimization in Statistical Package for the Social Sciences software. In order to overcome time-consuming computations and easy fall into a local minimum, Luo [12] proposed an improved particle swarm optimization (IPSO) algorithm. In the IPSO algorithm, the Cauchy mutations were used to ensure lager mutation steps and to fall into a local minimal. Pan [13] introduced vehicle classification standards and related technical characteristics, then analyzed advantages and applicability of different vehicle types, and proposed a vehicle selection method. However, there is no research on optimization model for multi-vehicletype structure in urban bus systems.

In this paper, an optimization model for multi-vehicletype structure in urban bus systems is presented. The proposed method was tested on real urban bus system. The obtained results have shown that proposed method provides better prediction and achieves better results than commonly used methods. The paper is organized as follows. In Section 2, a problem statement is explained, and all used assumptions and constraints are provided. The proposed model is presented in Section 3. In Section 4, we propose a method to solve the optimization model, while its verification by real urban bus system is exposed in Section 5. Lastly, a brief conclusion is given in Section 6.

\section{Problem Statement and Assumptions}

In this section, a scheduling problem in the multi-vehicle bus systems will be explained. The optimization of multi-vehicletype structure was based on some assumptions, which are listed in the following:

(i) Bus lines share the same terminal, or the terminals of each bus line are very close to each other, so it can be considered that all lines share the same terminal.

(ii) The parking function is provided.

(iii) All buses start from and end at the same terminal.

(iv) All buses belong to one bus company. (v) All bus lines have two fixed departure intervals, one for peak period and one for off-peak period, whereby the first one is smaller than the second one.

(vi) Average daily traffic is considered constant.

(vii) Average speed of buses is a constant value.

(viii) All base fares are equal.

(ix) There are $K$ bus lines.

(x) Work time of terminal is divided into $i$ periods with the same length.

(xi) There are $J$ available bus types.

In conventional bus operating, the dispatcher generally sets up fixed departure interval. However, sometimes a dynamic departure interval is chosen, which has its own advantages and disadvantages. Nonetheless, due to restrictions on cost in real bus systems, the dispatcher mostly chooses two fixed departure intervals based on different traffic volumes, departure interval in peak period, and departure interval in off-peak period. Likewise, in this paper two fixed departure intervals were selected so that they satisfy the following conditions: $2 \leqslant t_{g k}, t_{p k} \leqslant 20$, where $t_{g k}$ represents departure interval of bus line $k$ in peak period and $t_{p k}$ represents departure interval of bus line $k$ in off-peak period.

As already mentioned the work time of terminal was divided into $i$ intervals with the same length. The work time of the terminal is defined by $T=T_{l}-T_{e}$, where $T_{l}$ is the latest operating time of bus lines and $T_{e}$ is the earliest operating time of bus lines. Nevertheless, there are differences in operating distribution between bus lines. The number of passengers is also different on different lines. However, all bus lines should meet the volume demand, which is defined by

$$
Q_{i k} \leq \sum_{k=1}^{K} \sum_{j=1}^{J} n_{i k j} \times C_{j} \times \eta_{i k j},
$$

where $K$ is the number of bus lines, $J$ is the number of available bus types, $Q_{i k}$ represents the maximum passenger volume on bus line $k$ in time period $i, n_{i k j}$ denotes the number of $j$-type vehicles on line $k$ in time period $i, C_{j}$ is the passenger capacity of $j$-type vehicles, and $n_{i k j}$ represents expected passenger volume in $j$-type vehicles on line $k$ in time period $i$. Afterwards, the number of $j$-type buses, that is, $j$ type vehicles, on all lines in period $i$ is defined by

$$
N_{i j}=\sum_{k=1}^{K} n_{i k j} .
$$

Hence, the total number of $j$-type vehicles is defined by

$$
N_{j}=\max N_{i j} .
$$

Meanwhile, the total number of vehicles is defined by

$$
N=\sum_{j=1}^{J} N_{j}
$$


Moreover, the number of vehicles on line $k$ should satisfy the capacity condition:

$$
N_{k} \geq \operatorname{EVEN}\left(\frac{60 \cdot l_{k}}{\bar{V}_{g k} \times t_{g k}}\right),
$$

where $N_{k}$ is the total number of vehicles on line $k, l_{k}$ is the length of line $k$ in kilometers, and $\bar{V}_{g k}$ is the average speed of vehicles on line $k$ in peak period. Therefore, (4) can be rewritten as

$$
N=\sum_{j=1}^{J} N_{j}=\sum_{k=1}^{K} N_{k}
$$

\section{Optimization Model}

3.1. Bus Company Expense. The departure intervals were selected based on actual activities and passenger needs. If traffic volume is not considered, departure interval should meet the peak capacity demand for a single-type vehicle configuration, wherein vehicles of large or medium sizes are used. Therefore, the number of bus drivers would be strictly defined; thus, the human cost can be considered as a constant. Due to what was previously mentioned, human cost, employee benefits, and bonus were neglected in the bus company expense. On the other hand, operating and maintenance cost included repairs cost and fuel cost. In fact, the operating and maintenance cost is related to vehicle mileage. Therefore, the paper defines two cost types, production cost and vehicle mileage cost. Eventually, we analyzed three main parts of bus company expense: cost of vehicles utilization, operating and maintenance cost, and cost of carbon emission treatment.

The cost of vehicles utilization is defined by

$$
C_{c}=\sum_{j=1}^{J} N_{j} \times c_{j}
$$

where $c_{j}$ is the price of $j$-type vehicle. Subsequently, daily cost of vehicles utilizations is defined by

$$
C_{c d}=\frac{\sum_{j=1}^{J} N_{j} \times c_{j}}{d} .
$$

Furthermore, the operating and maintenance cost is defined by

$$
C_{y}=\sum_{k=1}^{K} \sum_{j=1}^{J} 2 \times l_{k} \times m_{k j} \times r_{j}
$$

where $m_{k j}$ is the number of departure times of $j$-type vehicles on bus line $k$ on daily base and $r_{j}$ is the unit operating cost of line $k$.
Moreover, the cost of carbon emission treatment is defined by

$$
\begin{aligned}
C_{E} & =\mu \sum_{k=1}^{K} \sum_{j=1}^{J} 2 \times l_{k} \times m_{k j} \times E_{j} \\
& =\mu \sum_{k=1}^{K} \sum_{j=1}^{J} 2 \times l_{k} \times m_{k j} \times \xi \times \frac{B_{j}}{100},
\end{aligned}
$$

where $\mu$ is the unit cost of carbon treatment, $E_{j}$ denotes the carbon emission, $\xi$ represents the fuel consumption per $100 \mathrm{~km}$ of $j$-type vehicles, and $B$ is the carbon emission factor.

The number of departure times of all lines on daily base is defined by

$$
M=\sum_{j=1}^{J} M_{j}=\sum_{j=1}^{J} \sum_{i=1}^{I} \sum_{k=1}^{K} m_{i k j}=\sum_{j=1}^{J} \sum_{i=1}^{I} \sum_{k=1}^{K} n_{i k j},
$$

where $M_{j}$ is the number of departure times of $j$-type vehicles on daily base. Thus, the bus company expense is defined by

$$
\begin{aligned}
C= & C_{c d}+C_{y}+C_{E} \\
= & \frac{\sum_{j=1}^{J} N_{j} \times c_{j}}{d}+\sum_{k=1}^{K} \sum_{j=1}^{J} 2 \times l_{k} \times m_{k j} \times r_{j} \\
& +\mu \sum_{k=1}^{K} \sum_{j=1}^{J} 2 \times l_{k} \times m_{k j} \times E_{j},
\end{aligned}
$$

where $d$ represents the length of bus operation life. However, the operating cost of various types of vehicles and the cost of carbon treatment are related to bus mileage. Due to differences in lines' length and number of vehicle types on each line, the minimal sum of operating and maintenance cost and cost of carbon emission treatment in time period $i$ is defined by

$$
\begin{aligned}
& \min \left(C_{y i}+C_{E i}\right)=\min \left(\sum_{k=1}^{K} \sum_{j=1}^{J} 2 \times l_{k} \times m_{k j} \times r_{j}\right. \\
& \left.+\mu \sum_{k=1}^{K} \sum_{j=1}^{J} 2 \times l_{k} \times m_{k j} \times E_{j}\right) .
\end{aligned}
$$

3.2. Bus Service Quality. In addition to the bus company expense, we analyzed the bus service quality. Due to fixed departure intervals and fixed operating time of lines, the bus service quality can be determined by daily passenger capacity provided by all lines. As mentioned before, the bus fare is equal for all lines; thus, the bus service quality can be expressed by total daily passenger capacity. Considering that average passenger volume is constant, greater capacity would provide higher bus service quality. Consequently, bus service quality is defined by

$$
S=p \sum_{k=1}^{K} \sum_{j=1}^{J} m_{k j} \times \eta_{j}
$$


where $p$ is the base fare and $\eta_{j}$ represents the expected passenger volume in $j$-type vehicles.

3.3. Multi-Vehicle-Type Model. The aim of this paper is to determine the total bus company expense and to calculate the bus service quality and, finally, to optimize the bus system in order to reduce the cost and to increase the quality. In fact, different interest groups have different opinion on weight coefficients $\alpha$ and $\beta$; for instance, bus companies profit if $\alpha$ is much larger than $\beta$, while the opposite solution is more convenient for bus passengers; thus, we did not specify their values here. Therefore, the multi-vehicle-type configuration model can be described by

$$
\begin{aligned}
G & =\max (-\alpha C+\beta S)=\max \left\{-\alpha\left[\frac{\sum_{j=1}^{J} N_{j} \times c_{j}}{d}\right.\right. \\
& +\sum_{k=1}^{K} \sum_{j=1}^{J} 2 \times l_{k} \times m_{k j} \times r_{j} \\
& \left.+\mu \sum_{k=1}^{K} \sum_{j=1}^{J} 2 \times l_{k} \times m_{k j} \times E_{j}\right] \\
& \left.+\beta p \sum_{k=1}^{K} \sum_{j=1}^{J} m_{k j} \times \eta_{j}\right\}
\end{aligned}
$$

where $C$ represents the bus company expense, $S$ denotes the bus service quality, $\alpha$ is the weight coefficient of bus company expense, and $\beta$ is the weight coefficient of bus service quality. Obviously, different stakeholders have different opinions on $\alpha$ and $\beta$. In this study, Delphi method was used to determine the numerical value of $\alpha$ and $\beta$. According to experts on public transport, the specific values represent the average values.

\section{The Markov Method}

The proportion of bus vehicle types on regular bus lines is affected by various random factors. The nonaftereffect property of vehicle structure can satisfy the analysis condition of Markov method; thus, the development trend of vehicle structure can be analyzed and predicted by Markov method.

However, it is difficult to obtain the transfer probability $P_{a b}$ of $a$-type vehicle to $b$-type vehicle by Markov method. Namely, the important assumption in Markov method is that the state transition matrix does not change with time, while the structure of different bus vehicle structures changes in an unbalanced time series. Therefore, in order to overcome this problem, we established a multi-vehicle-type structure prediction model based on Markov method and alternative relation. Hence, for bus line $k$, one has the following:

(a) The proportion of $j$-type buses in time period $i$ is sum of $f_{1}(i), f_{2}(i), \ldots, f_{J}(i)$ :

$$
\sum_{j=1}^{J} f_{j}(i)=1
$$

(b) $a$ and $b$ are different vehicle types contained in $J$ vehicle types, and $a=1,2, \ldots, J$. From time period $i$ to time period $(i+1)$, the proportion of $a$-type and $b$-type is $\Delta f_{a b}(i)$, and

$$
f_{j}(i)=\sum_{j=1}^{J} \Delta f_{a b}(i)
$$

(c) In time period $(i+1)$ the proportion of $a$-type is translated from 1 to $J$ types in time period $i$; therefore, it can be defined by

$$
f_{a}(i+1)=\sum_{b=1}^{J} \Delta f_{a b}(i) .
$$

(d) There is no correlation between proportion of $a$-type and $b$-type acquisition, and the proportion of $a$-type is only related to the proportion of all types in the last time period $f_{a}(i)(i+1)$.

(e) $\Delta f_{a b}(i)$ from time period $i$ to time period $(i+1)$ is proportional to $f_{a}(i)$ and $f_{b}(i)$ :

$$
\Delta f_{a b}(i)=\Delta G_{a b}(i) \times f_{a}(i) \times f_{b}(i),
$$

where $G_{a b}$ is the scale factor whose value determines the value of $\Delta f_{a b}(i)$.

(f) According to the above assumptions, from (17) and (19) we get

$$
f_{b}(i)=\sum_{a=1}^{J} G_{a b} \times f_{a}(i) \times f_{b}(i) .
$$

Similarly, from (18) and (19), we get the following:

$$
f_{a}(i+1)=\sum_{b=1}^{J} G_{b a} \times f_{b}(i) \times f_{a}(i) .
$$

Since $f_{a}(i) \neq 0$, the optimization model can be obtained by (20) and (21):

$$
\begin{aligned}
& \sum_{a=1}^{J} G_{a b} \times f_{a}(i)=1, \\
& \sum_{b=1}^{J} G_{b a} \times f_{b}(i)=\frac{f_{a}(i+1)}{f_{a}(i)} .
\end{aligned}
$$

(g) Using the linear regression analysis in SPSS software, we first estimate the parameter $G_{a b}$; namely, (22) is used to estimate the initial $G_{0}$. Then, the objective function is selected by

$$
\begin{aligned}
R_{s} & =\frac{1}{I} \sum_{i=1}^{I} \sum_{a=1}^{J}\left\{\left[1-\sum_{b=1}^{J} G_{a b} \times f_{b}(i)\right]^{2}\right. \\
& \left.+\left[\frac{f_{a}(i+1)}{f_{a}(i)}-\sum_{a=1}^{J} G_{b a} \times f_{a}(i)\right]^{2}\right\} .
\end{aligned}
$$


TABLE 1: Related information on bus lines.

\begin{tabular}{lcc}
\hline Line & A & B \\
\hline$L[\mathrm{~km}]$ & 11 & 8 \\
Time range $[\mathrm{h}]$ & 14 & 12 \\
$N_{k}$ & 14 & 10 \\
Vehicle type & Large & Large \\
$C$ & 110 & 110 \\
$t_{g}[\mathrm{~min}]$ & 5 & 6 \\
$t_{p}[\mathrm{~min}]$ & 8 & 8 \\
Average vehicle speed in peak period $[\mathrm{km} / \mathrm{h}]$ & 19 & 16 \\
Average vehicle speed in off-peak period $[\mathrm{km} / \mathrm{h}]$ & 22 & 20 \\
Line character & Commuter service & Shopping service
\end{tabular}

Afterwards, the target condition of planning solutions is set; therefore, we get the minimum of $R_{s}$ :

$$
\begin{gathered}
\min R_{s}=\frac{1}{I} \sum_{i=1}^{I} \sum_{a=1}^{J}\left\{\left[1-\sum_{b=1}^{J} G_{a b} \times f_{b}(i)\right]^{2}\right. \\
\left.+\left[\frac{f_{a}(i+1)}{f_{a}(i)}-\sum_{a=1}^{J} G_{b a} \times f_{a}(i)\right]^{2}\right\}
\end{gathered}
$$

where $\sum_{a=1}^{J} f_{a}(i)=1$. Lastly, the optimal solution $G_{a b}$ can be obtained by (23)-(24).

\section{Model Verification}

Model verification was conducted with two bus lines that had the same terminal in Jilin. The base fare of both lines was 1 yuan. According to the traffic system, Line A connected several schools and enterprises; thus, Line A mainly serviced the commuter volume. On the other hand, Line B connected several shopping centers and residential areas; thus, Line B mainly serviced the shopping volume. All related information on bus lines is presented in Table 1 . In addition, the maximum hourly passenger volume of both lines and the sum of maximum hourly passenger volumes are presented in Figure 1.

The operating time of both lines was 14 hours, and we divided that period into 14 periods of the same length. The technical parameters of vehicles are presented in Table 2.

According to the distribution of hourly passenger volume of Line A and Line B presented in Figure 1, it can be noticed that from 7:30 to $8: 30$ traffic volume was the highest. If we assume that expected passenger volume on Line $\mathrm{A}$ in that time period is 1 , according to (5), the capacity of Line A is $Q_{13}=1260>925$. The analysis of results has proven that Line A satisfies traffic volume condition in 7:30 to 8:30 period. Similarly, the capacity provided by Line $B$ is $Q_{23}=720>285$.

Subsequently, the analysis has shown that Line A also meets the traffic volume condition in mentioned period, but with a huge waste of resources. According to the results obtained by MATLAB software, it is reasonable to arrange 20 buses for two lines. If we assume that numbers of large vehicles, medium vehicles, and small vehicles are $N_{1}, N_{2}$, and $N_{3}$, respectively, then the best model configuration obtained

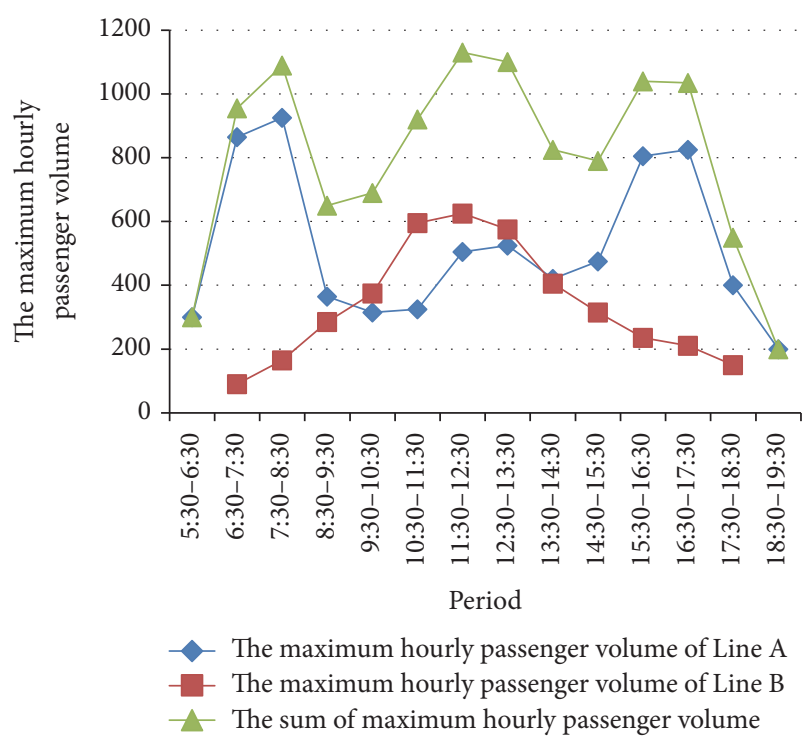

FIGURE 1: The maximum hourly passenger volumes on Lines A and $B$ and their sum.

TABLE 2: Technical parameters of vehicles.

\begin{tabular}{lcc}
\hline \multirow{2}{*}{ Index } & \multicolumn{2}{c}{ Vehicle types } \\
& Large & Medium \\
\hline$C_{j}$ & 80 & 50 \\
$C_{c}$ [yuan] & 750000 & 550000 \\
$d[$ day] & 2920 & 2920 \\
$C_{y}[$ yuan $\cdot \mathrm{km}]$ & 2.86 & 2.07 \\
$E_{j}[\mathrm{~g} / \mathrm{km}]$ & 62 & 57 \\
$r_{j}[$ yuan $\cdot \mathrm{g} / \mathrm{km}]$ & 2.85 & 2.43 \\
$\mu[$ yuan $/ \mathrm{km}]$ & 0.00005 & 0.00005 \\
$\xi[\mathrm{kg} \mathrm{CO} / \mathrm{L}]$ & 2.77 & 2.77 \\
$\mathrm{~B}[\mathrm{~L}]$ & 33 & 26 \\
Length of vehicle $[\mathrm{m}]$ & 10.5 & 8.5 \\
Fuel type & Diesel & Diesel \\
\hline
\end{tabular}

by SPSS software is $\left(N_{1}, N_{2}, N_{3}\right)=(8,12,0)$, while the optimal solutions for $\alpha$ and $\beta$ obtained by Delphi method are $\alpha=0.9$ and $\beta=0.1$. The comparison of original vehicle 
TABLE 3: Comparison of original vehicle configuration and optimal vehicle configuration obtained by the proposed model.

\begin{tabular}{|c|c|c|c|c|c|}
\hline \multirow{2}{*}{ Parameter } & \multicolumn{3}{|c|}{ Original vehicle configuration } & \multirow{2}{*}{ Proposed model } & \multirow{2}{*}{ Value added } \\
\hline & Line A & Line B & Sum & & \\
\hline$N$ & 14 & 10 & 24 & 20 & -4 \\
\hline$C_{c d}$ [yuan] & 3595.890411 & 2568.49315 & 6164.38356 & 4315.068493 & -1849.3151 \\
\hline$C_{y}$ [yuan] & 8557.12 & 2782.08 & 11339.2 & 12357.6 & 1018.4 \\
\hline$C_{E}$ [yuan] & 136.74936 & 61.42752 & 198.17688 & 172.93958 & -25.2373 \\
\hline$S$ [yuan] & 10880 & 6720 & 17600 & 13010 & -4590 \\
\hline G & -33111.16736 & -10462.061 & -43573.228 & -13863.09122 & 29710.137 \\
\hline
\end{tabular}

configuration and optimal vehicle configuration obtained by the proposed model is presented in Table 3.

According to the results presented in Table 3, it can be concluded that the proposed model can effectively reduce the bus company expense and improve the bus service quality.

\section{Conclusion}

An optimization model intended for multi-vehicle-type structure in urban bus systems is presented. The model is developed based on set of premises and assumptions. The multi-vehicle-type structure with two bus lines that have the same terminal was considered. Moreover, all buses had unified schedule in order to reduce the bus company expense and to improve the bus service quality. Furthermore, the impact of carbon treatment on the bus company expense was also analyzed. In addition, we analyzed the bus service quality and it was concluded that it can be measured by passenger capacity provided by all lines. The comparison of proposed model and original model was conducted according to the bus company expense and bus service quality, and it was concluded that proposed model can effectively reduce the bus company expense and improve the bus service quality.

\section{Conflicts of Interest}

The authors declare that they have no conflicts of interest.

\section{Acknowledgments}

The authors thank Jilin Tongtai Traffic Science and Technology Consulting Co., Ltd., for bus lines data. The work was supported by the National Natural Science Foundation of China (Grant no. 51378237) and the National Natural Science Foundation of China (Grant no. 51608224).

\section{References}

[1] J. A. Ferland and P. Michelon, "The vehicle scheduling problem with multiple vehicle types," Journal of the Operational Research Society, vol. 39, no. 6, pp. 577-583, 1988.

[2] A. Ceder, "Transit vehicle-type scheduling problem," Transportation Research Record, no. 1503, pp. 34-38, 1995.

[3] K. Ker, H. F. Kuo, and M. Schondeld, "Optimal mixed bus fleet for urban operations," Transportation Research Record, vol. 1503, pp. 39-48, 1995.

[4] P. D. Site and F. Filippi, "Bus service optimisation with fuel saving objective and various financial constraints," Transportation
Research Part A: Policy and Practice, vol. 35, no. 2, pp. 157-176, 2001.

[5] F. Zhu, W. Deng, and L. Ge, "Study on equipment method of bus line capacity," Journal of Transportation Engineering and Information, vol. 3, no. 1, pp. 93-97, 2005.

[6] N. Kliewer, T. Mellouli, and L. Suhl, "A time-space network based exact optimization model for multi-depot bus scheduling," European Journal of Operational Research, vol. 175, no. 3, pp. 1616-1627, 2006.

[7] L. dell'Olio, A. Ibeas, and F. Ruisánchez, "Optimizing bus-size and headways in transit networks," Transportation, vol. 39, no. 2, pp. 449-464, 2012.

[8] J. Sun, Y. Xu, and Z. Peng, "Timetable optimization for single bus line based on hybrid vehicle size model," Journal of Traffic and Transportation Engineering, vol. 2, no. 3, pp. 179-186, 2015.

[9] Y. Huang, Z. Wang, Z. Li, and C. Zhu, "Coordinated optimization of bus and departure intervals in multi-vehicle bus system," Journal of Changsha University of Science and Technology (Natural Science), vol. 13, no. 3, pp. 37-41, 2016.

[10] H. Xiong and L. Hu, "Dynamic vehicle routing problem with multiple vehicle type and its genetic algorithm," Systems Engineering, vol. 27, no. 10, pp. 21-24, 2009.

[11] M. Huo, "Study on city bus capacity," Chongqing Jiaotong University, Chongqing, China, 2009.

[12] B. Luo, "Study on multi-depots and multi-vehicles vehicle scheduling problem based on improved particle swarm optimization," Computer Engineering and Applications, vol. 50, no. 7, pp. 251-253, 2014.

[13] X. Pan, "Research on bus capacity structure configuration optimization model of urban transit," Jilin University, Changchun, China, 2016. 


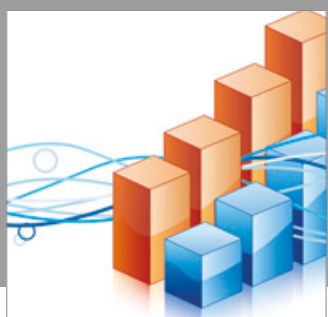

Advances in

Operations Research

vatersals

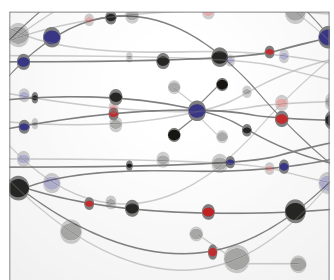

\section{The Scientific} World Journal
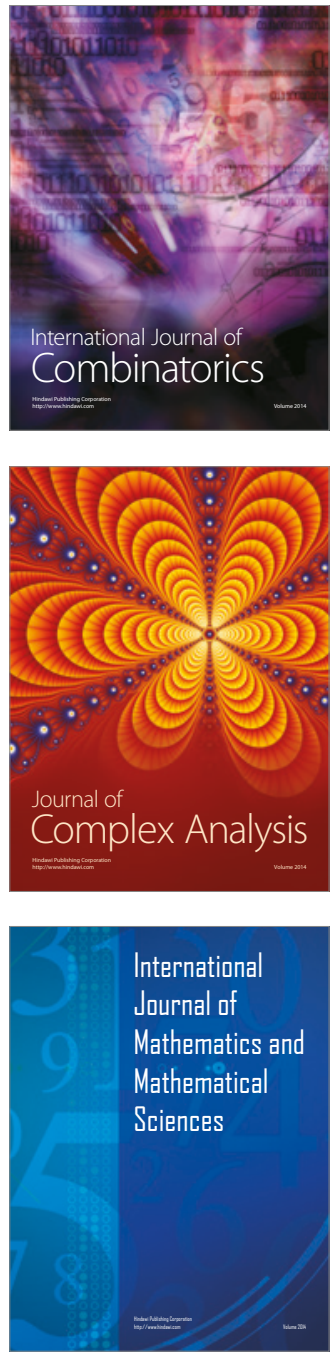
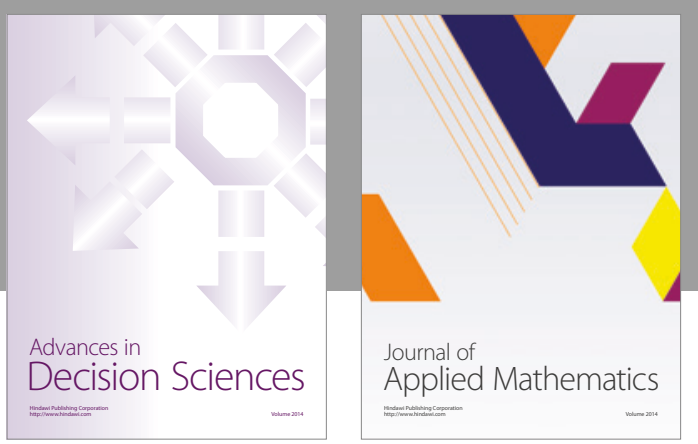

Algebra

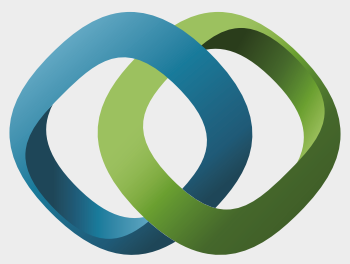

\section{Hindawi}

Submit your manuscripts at

https://www.hindawi.com
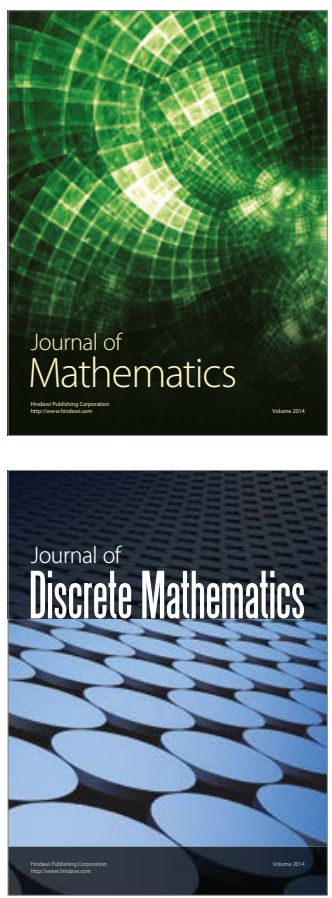

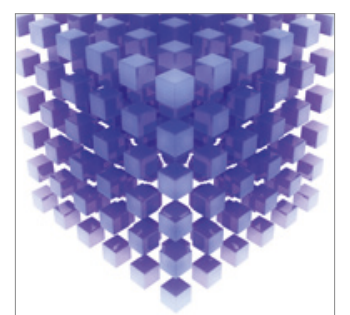

Mathematical Problems in Engineering
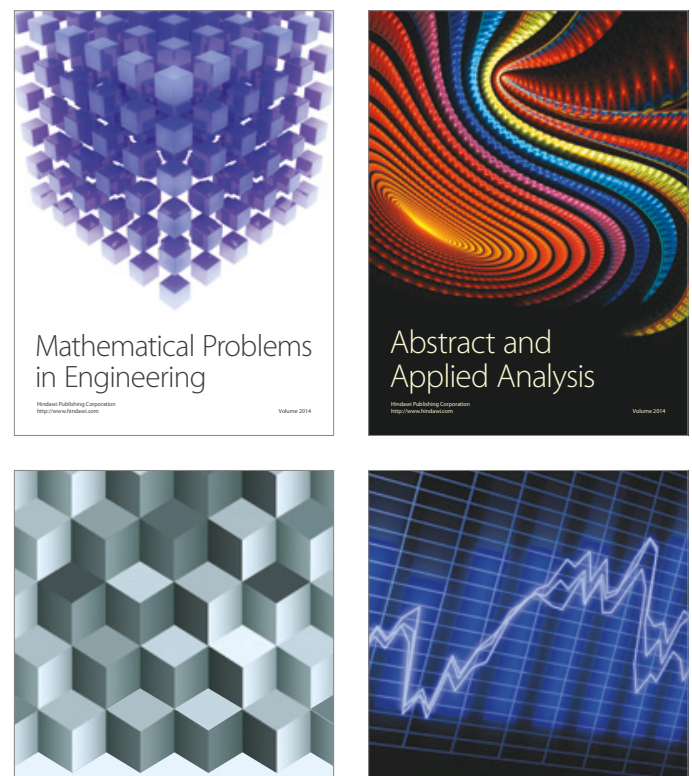

Journal of

Function Spaces

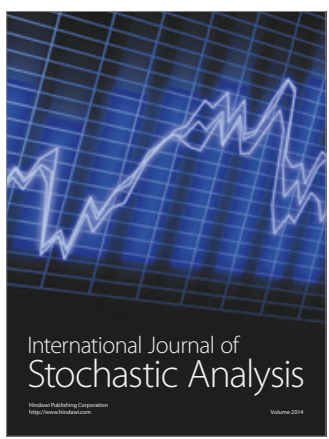

Probability and Statistics
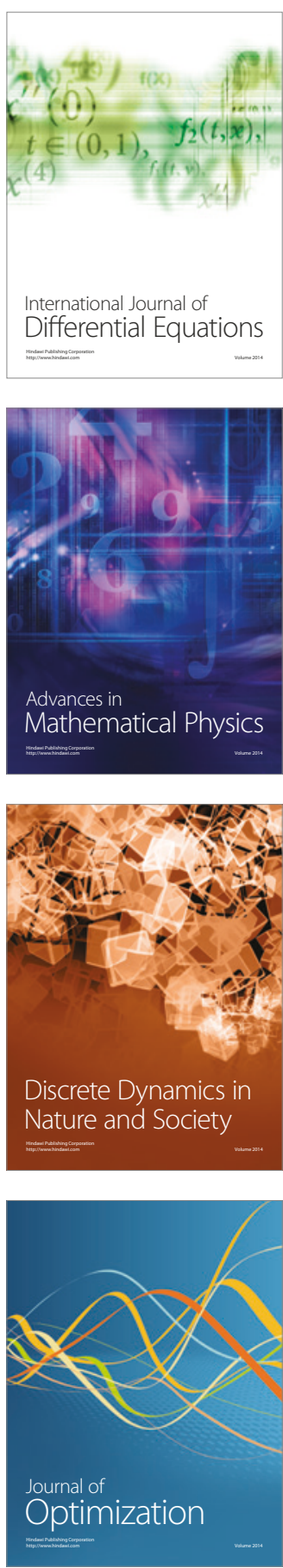